\title{
Influence of the reaction field on the nature of the phase transition in the Potts model
}

\author{
R Dekeyser $\dagger$, A Maritan $\ddagger$ and A L Stella $\$$ \\ $\uparrow$ Instituut voor theoretische fysika, K U Leuven, B-3030 Heverlee, Belgium \\ ¥International School for Advanced Studies (SISSA), Trieste, and INFN, Sezione di \\ Trieste, Italy \\ $\S$ Istituto di Fisica e Unità GNSM del CNR dell' Universita di Padova, Italy and Inter- \\ national School for Advanced Studies (SISSA), Trieste, Italy
}

Received 6 August 1982

\begin{abstract}
It is shown how Onsager's reaction field may be incorporated in mean-field-like calculations for the $q$-state Potts model. Its influence on the nature of the phase transition is considered both in a one-site and a two-site cluster approximation. Considerable improvement is obtained for $q_{c}(d)$, the value which separates first- and second-order transitions. The way in which $q_{c}(d)$ is modified when a second-neighbour interaction is added to the ferromagnetic nearest-neighbour one is also studied.
\end{abstract}

\section{Introduction}

The nature of the phase transition in the $q$-state Potts model has posed a special problem since the introduction of the model (Potts 1952, Wu 1982, Nienhuis et al 1981). Exact arguments (Baxter 1973) in $d=2$, e.g., show that a first-order transition occurs for $q>4$ and a second order one for $q \leqslant 4$. In general, there should exist a function $q_{\mathrm{c}}(d)$, such that in $d$ dimensions the change from second to first order occurs at $q=q_{\mathrm{c}}(d)$. Up to now no exact expression for $q_{\mathrm{c}}(d)$ is known, although from a combination of several approximate methods one has obtained a rather precise idea of the general behaviour of this function (Wu 1982, Nienhuis et al 1981).

On the other hand, $q_{\mathrm{c}}(d)$ is expected to be a non-universal quantity; as a consequence it could be strongly sensitive to modifications of the Hamiltonian, such as the inclusion of second-neighbour ferromagnetic or antiferromagnetic interactions (Fucito and Parisi 1981). Evidence for such effects has been obtained recently by Monte Carlo simulations (Fucito and Vulpiani 1982), but an analytical way of approaching this problem, at least qualitatively, has been lacking up to now.

Simple position space renormalisation calculations (Burkhardt et al 1976) predict a second-order transition for all $q$, and only the introduction of vacancies in the model allows the calculation of a non-trivial estimate for $q_{c}(d)$ (Nienhuis et al 1979, 1981). A straightforward molecular field approximation (Kihara et al 1954, Mittag and Stephen 1974) predicts on the contrary a first-order transition for $q>2$ and for all $d$. It is thus obvious to look for an improvement on this approximation that might eventually yield a more realistic estimate for $q_{\mathrm{c}}(d)$, and some insight into its dependence on the type of Hamiltonian. The physically most promising way for correcting the simplifications of the molecular field approximation is to take account of Onsager's 
reaction field (Onsager 1936, Brout and Thomas 1967, Dekeyser and Halzen 1969). In the framework of magnetic systems, this field takes into account the influence of a given spin on its neighbours, in the sense that the thermal average of a neighbouring spin will be allowed to depend on the actual orientation of the central spin. We now propose to investigate the influence of this reaction field on the nature of the phase transition in the $q$-state Potts model.

In $\S 2$ we briefly summarise the formulae of relevance to the Potts model and its molecular field approximation. In $\$ 3$ we derive an expression for the reaction field in the nearest-neighbour case, and in $\$ 4$ we determine its consequences on the nature of the phase transition. Section 5 is devoted to the discussion of the effect on $q_{c}(d)$ of a further-neighbour interaction.

\section{Description of the model and molecular field approximation}

In the $q$-state Potts model a variable $s_{i}$ is associated with each site $i$ of a regular lattice; it may assume the $q$ values $s_{i}=0,1, \ldots, q-1$. We consider the Hamiltonian

$$
\begin{aligned}
H_{h} & =-J \sum_{\langle i j\rangle} \delta_{s_{i,}, s_{i}}-\sum_{k=0}^{q-1} \sum_{i} h_{k i} \delta_{s_{i, k}, k} \\
& =-\sum_{k=0}^{q-1}\left(J \sum_{\langle i j\rangle} \delta_{s_{i}, k} \delta_{s_{,}, k}+\sum_{i} h_{k i} \delta_{s_{i}, k}\right) .
\end{aligned}
$$

The first sum runs over all nearest-neighbour pairs $\langle i j\rangle$. The fields $h_{k i}$ will eventual'y be equated to zero, since our main interest lies in the Hamiltonian $H_{0}$. Below a certain temperature condensation is expected to occur into e.g. the state $s=0$, such that the thermal averages of the state occupations are given by

$$
\begin{aligned}
& p_{0} \equiv\left\langle\delta_{s_{i}, 0}\right\rangle=[1+(q-1) m] / q, \\
& p_{n} \equiv\left\langle\delta_{s_{i, n}}\right\rangle=(1-m) / q \quad(n \geqslant 1) .
\end{aligned}
$$

$m$ is the order parameter of the model. In principle the $p_{k}$ may be calculated from the partition function $Z$ of the system by

$$
\left\langle\delta_{s, k}\right\rangle=\left.\frac{1}{\beta} \frac{\partial \log Z}{\partial h_{k i}}\right|_{h=0} .
$$

In the standard mean field approximation, one assumes that the $\delta_{s, k}$ at the $z$ nearest-neighbour lattice sites of any site $i$ may be replaced in (1) by their average values according to (2). The one-particle partition function (for $h=0$ ) is then given by

$$
Z_{1}=\sum_{k=0}^{q-1} \exp \left(K z p_{k}\right)
$$

and

$$
\left\langle\delta_{s_{i}, k}\right\rangle=Z_{1}^{-1} \exp \left(K z p_{k}\right),
$$

where $K=\beta J$. With the assumption (2), this leads to the standard mean field equation

$$
m=\frac{q\left\langle\delta_{s_{1}, 0}\right\rangle-1}{q-1}=\frac{\mathrm{e}^{K z m}-1}{\mathrm{e}^{K z m}+q-1} \equiv F_{1}(K z m) .
$$


One easily verifies that $F_{1}(x)$ is a monotonously increasing function of $x$, such that its inverse function $F_{1}^{-1}$ is uniquely defined. This allows us to write (5) in the alternative form

$$
g(m) \equiv F_{1}^{-1}(m)-K z m=0 .
$$

It is straightforward to show that, up to a constant factor $q /(q-1), g(m)$ is the derivative with respect to $m$ of $\beta f$, where $f$ is the $m$-dependent free energy function per lattice site in this simple mean field approximation, as calculated e.g. by Mittag and Stephen (1974). The equilibrium magnetisation $m_{0}$ is then the root of (6) at which $\beta f$ has an absolute minimum; this implies that necessarily $g^{\prime}\left(m_{0}\right)$ has to be positive. The negative second-order term in the Taylor expansion

$$
g(m)=(q-K z) m-\frac{1}{2} q(q-2) m^{2}+\ldots
$$

leads directly to the conclusion of a first-order transition for $q>2$. For $q<2$ this formula suggests a first-order transition into a state with negative $m$. This should in general be excluded as an artifact of the approximation, since the ground state of (1) has $m=1$. This aspect of the mean field approximation has been discussed before (Harris et al 1975, Priest and Lubensky 1976), and in the remaining part of the paper we will assume $0 \leqslant m \leqslant 1$, in the hope that a full theory would indeed exclude thermodynamic states with $m<0$.

\section{The reaction field in the Potts model}

In order to derive the reaction field for the Potts model, we repeat the calculation of $\S 2$, but in writing down the one-particle partition function for $s_{i}$, we will use the conditional probabilities for the $\delta_{s_{i, k}}$ :

$$
Z_{1}=\sum_{k=0}^{q-1} \exp \left(K z p_{k}^{c}\right)
$$

where

$$
\left.p_{k}^{c} \equiv\left\langle\delta_{s_{,}, k}\right\rangle\right|_{s_{i}=k}
$$

is the probability for finding $s_{j}=k$ when we know that $s_{i}=k$ at the neighbouring site $i$.

In order to calculate these $p_{k}^{c}$, we define for a nearest-neighbour pair $(i, j)$ the susceptibilities

$$
\left.\chi_{k} \equiv \frac{\partial\left\langle\delta_{s_{i}, k}\right\rangle}{\partial h_{k j}}\right|_{h=0}=\beta\left(\left\langle\delta_{s_{v}, k} \delta_{s, k}\right\rangle-\left\langle\delta_{s_{\imath}, k}\right\rangle\left\langle\delta_{s, k}\right\rangle\right) .
$$

The last equation, a special case of the fluctuation-dissipation theorem, is obvious from (3). Furthermore, we have

$$
\left\langle\delta_{s_{i}, k} \delta_{s_{j}, k}\right\rangle=\left.\left\langle\delta_{s_{i}, k}\right\rangle\left\langle\delta_{s_{r}, k}\right\rangle\right|_{s_{t}=k}=p_{k} p_{k}^{c} .
$$

Combining (10) and (11) gives

$$
p_{k}^{c}=p_{k}+\chi_{k} / \beta p_{k} .
$$


$\chi_{k}$ may be determined approximately as follows:

$$
\chi_{k} \simeq \sum_{k^{\prime}} \frac{\partial\left\langle\delta_{s_{i, k}}\right\rangle}{\partial\left\langle\delta_{s_{i, k},}\right\rangle} \frac{\partial\left\langle\delta_{s, k^{\prime}}\right\rangle}{\partial h_{k j}},
$$

where we have neglected the dependence of $\left\langle\delta_{s_{1}, k}\right\rangle$ on $h_{k j}$ through intermediate lattice sites. Since $j$ is one of the $z$ neighbours of site $i$, we may use our previous result $(4 b$ ) to obtain a first-order correction. This yields

$$
\partial\left\langle\delta_{s_{1}, k}\right\rangle / \partial\left\langle\delta_{s_{,}, k^{\prime}}\right\rangle=K p_{k}\left(\delta_{k, k^{\prime}}-p_{k^{\prime}}\right)
$$

and

$$
\partial\left\langle\delta_{s_{i}, k^{\prime}}\right) / \partial h_{k j}=\beta p_{k}\left(\delta_{k, k^{\prime}}-p_{k^{\prime}}\right)
$$

and thus

$$
\chi_{k} \simeq \beta K p_{k}^{2}\left(\left(1-p_{k}\right)^{2}+\sum_{k^{\prime} \neq k} p_{k^{\prime}}^{2}\right) .
$$

Substituting now (2) and (16) in (12), we get

$$
\begin{aligned}
& p_{0}^{c}=\{[1+(q-1) m] / q\}\left[1+(K / q)(1-m)^{2}(q-1)\right], \\
& p_{n}^{c}=[(1-m) / q]\left\{1+(K / q)\left[q\left(1+m^{2}\right)-(1-m)^{2}\right]\right\} \quad(n \geqslant 1) .
\end{aligned}
$$

Using now these conditional probabilities in (8), we easily obtain the selfconsistency equation, which can be written in the form

$$
m=F_{1} \llbracket K z m\{1-(K / q)(1-m)[(3-q)+(q-1) m]\} \rrbracket,
$$

where $F_{1}(x)$ was defined in (5). Whereas the term $K z m$ has its origin in the usual molecular field exerted on site $i$ by its $z$ neighbours, the remaining term in the argument of $F_{1}$ describes the correction due to the reaction field. Instead of $(6)$, we now have the following improved approximation for the derivative of the free energy with respect to $m$ :

$$
\begin{aligned}
g_{1}(m) & =F_{1}^{-1}(m)-K z m\{1-(K / q)(1-m)[(3-q)+(q-1) m]\} \\
& =\left(q-K z+\left(K^{2} z / q\right)(3-q)\right) m-\frac{1}{2}(q-2)\left(q-4 K^{2} z / q\right) m^{2}+\ldots
\end{aligned}
$$

\section{Nature of the phase transition}

Since we assume $m \geqslant 0$, we expect to find a second-order phase transition when the expansion of the free energy in powers of the order parameter

$$
f(m)=A_{2} m^{2}+A_{3} m^{3}+\ldots
$$

has the properties $A_{2}=0, A_{3}>0$. This means that, apart from the possibility of extrema at higher $m$-values caused by negative higher-order terms, we may determine the critical value $q_{\mathrm{c}}$ above which no second-order transition will exist, by solving simultaneously $A_{2}=0$ and $A_{3}=0$. Since $g(m)$ is proportional to the derivative of $\beta f(m)$, this amounts to

$$
\begin{aligned}
& q-K z+\left(K^{2} z / q\right)(3-q)=0, \\
& q-\left(4 K^{2} z / q\right)=0
\end{aligned}
$$


with the solution

$$
q_{\mathrm{c} 1}=7-2 \sqrt{z}
$$

We know (Wu 1982) that, at least for $d=2, q_{c}$ is independent of the lattice, and thus $q_{\mathrm{c}}(d)$ should not depend directly on $z$. Therefore, we will limit ourselves to the lattice type that can be most easily extended to general dimensions, i.e. the hypercubic lattice with $z=2 d$. We then obtain

$$
q_{c 1}(d)=7-2 \sqrt{2 d}
$$

This determination of $q_{\mathrm{c}}$ is a considerable improvement on the poor mean field prediction $q_{\mathrm{c}}=2$ for all $d$.

A natural way for trying to obtain even better estimates for $q_{\mathrm{c}}(d)$ is to make a finite cluster calculation instead of the single-site calculation (8). The interactions inside the cluster have to be considered exactly, whereas the interaction between the cluster and environment may be treated in the mean field sense, again corrected by reaction field considerations. Let us take e.g. the example of a simple two-site cluster, in which each site has $z-1$ remaining neighbours, to be treated in a mean field sense. The partition function of this cluster may be written as

$Z_{2}=\sum_{k_{1}=0}^{q-1}\left(\exp \left\{K\left[1+2(z-1) p_{k_{1}}^{c}\right]\right\}+\sum_{k_{2} \neq k_{1}} \exp \left[K(z-1)\left(p_{k_{1}}^{c}+p_{k_{2}}^{c}\right)\right]\right)$.

Using (17), this leads us to the mean field equation

$$
m=F_{2} \llbracket K(z-1) m\{1-(K / q)(1-m)[(3-q)+(q-1) m]\} \rrbracket
$$

where

$$
F_{2}(x)=\frac{\mathrm{e}^{K+2 x}+(q-2) \mathrm{e}^{x}-\left(\mathrm{e}^{K}+q-2\right)}{\mathrm{e}^{K+2 x}+2(q-1) \mathrm{e}^{x}+(q-1)\left(\mathrm{e}^{K}+q-2\right)}
$$

and to the next approximation for $q(m)$ :

$$
g_{2}(m)=F_{2}^{-1}(m)-K(z-1) m\{1-(K / q)(1-m)[(3-q)+(q-1) m]\} .
$$

The function $q_{\mathrm{c} 2}(d=z / 2)$ for this two-site cluster may again be obtained by imposing the simultaneous vanishing of the coefficients of $m$ and $m^{2}$ in the series expansion of $g_{2}(m)$. This leads to the equations

$$
\begin{aligned}
& q \xi=K(z-1)[1-(K / q)(3-q)], \\
& 3 \xi^{2}-2 \xi^{3}=4 q^{-2}(z-1) K^{2},
\end{aligned}
$$

where

$$
\xi=\left(\mathrm{e}^{K}+q-1\right) /\left(2 \mathrm{e}^{K}+q-2\right) .
$$

These equations have to be solved numerically. The results are shown in figure 1 , together with $q_{\mathrm{c} 1}(d)$, and they are compared with the curve that is expected to represent the true values (Nienhuis et al 1981). We have also verified numerically that both $g_{1}(m)$ and $g_{2}(m)$ describe bona-fide second-order phase transitions for $q<q_{\mathrm{c} 1}$ and $q<q_{\mathrm{c} 2}$, respectively; in both cases there is only one minimum for the free energy for $m>0$. 


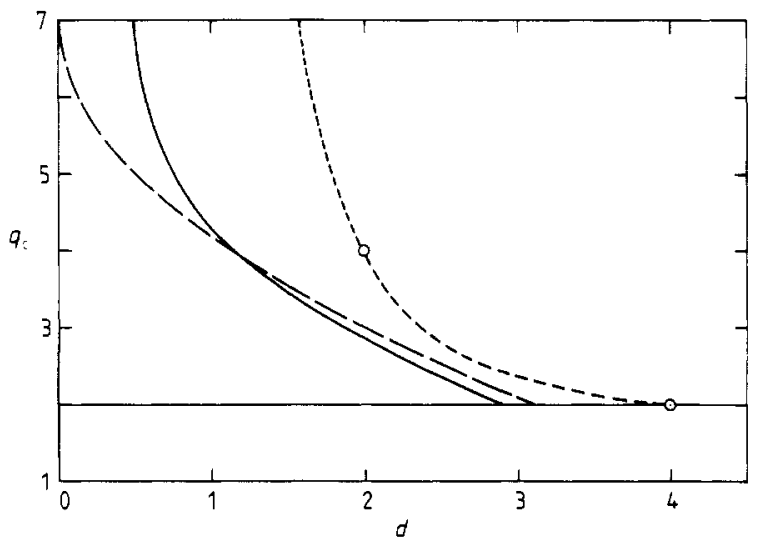

Figure 1. Values for $q_{c}(d)$ as obtained from a one-site (- - - ) and a two-site ( $\left.-\longrightarrow\right)$ approximation. $q_{c}(d)=2$ is the mean field result. The exactly known values are marked by dots, and the line :- - --' is a qualitative sketch of the expected true values.

\section{Dependence of $q_{\mathrm{c}}$ on further-neighbour interactions}

Our approximation scheme with reaction field corrections can also be used to study, at least qualitatively, the dependence of $q_{\mathrm{c}}$ on modifications of the Hamiltonian (1). The non-universal character of $q_{\mathrm{c}}$ has recently been stressed by Fucito and Parisi (1981); indeed, Monte Carlo simulations in three dimensions (Fucito and Vulpiani 1982) seem to indicate that $q_{\mathrm{c}}$ can become larger when a small antiferromagnetic further-neighbour interaction is included in (1). Monte Carlo studies have also shown that for a triangular lattice with two- and three-body interactions, $q_{\mathrm{c}}$ may depend on the coupling constants (Saito 1982).

Let us indicate by $J^{\prime}$ (and $L=\beta J^{\prime}$ ) the strength of a second-neighbour coupling to be added to (1). The standard mean-field-like calculation sketched in $\S 2$ may be repeated in this case, leading to a $g$-function identical to (7), except for the fact that $K z$ should be replaced by $\left(K z+L z^{\prime}\right)$, with $z^{\prime}$ being the number of second neighbours. This implies that the standard mean field treatment still leads to $q_{\mathrm{c}}=2$, independent of $L$. The presence of $L$, however, can modify $q_{\mathrm{c}}$ in a mean field calculation with reaction field corrections.

This calculation runs parallel to the one presented in $\S 3$. Due to the additional interactions with $z^{\prime}$ second neighbours, the single-spin partition function will now be of the form

$$
Z_{1}=\sum_{k=0}^{q-1} \exp \left(z K p_{k}^{c}+z^{\prime} L p_{k}^{\prime c}\right)
$$

where

$$
\left.p_{k}^{\prime c} \equiv\left\langle\delta_{s_{j}, k}\right\rangle\right|_{s_{i}=k},
$$

where $j$ is a second neighbour of $i . p_{k}^{\prime c}$ is the probability of finding the $j$ th spin in the $k$ th state, if we know that its second neighbour $i$ is in the same state. Of course $p_{k}^{\prime c}$ will not coincide with $p_{k}^{c}$. Indeed, a calculation similar to the one performed in $\S 2$ leads to the result (17) for $p_{k}^{c}$, and for $p_{k}^{\prime c}$ to a formula analogous to (17), but with $K$ 
replaced by $L$. The $g$-function obtained from (28) is then given by

$$
\begin{aligned}
g_{1}(m)=F_{1}^{-1}(m)-K z m & \{1-(K / q)(1-m)[(3-q)+(q-1) m]\} \\
-L z^{\prime} m & \{1-(L / q)(1-m)[(3-q)+(q-1) m]\}
\end{aligned}
$$

and the conditions determining $q_{\mathrm{c}}$ become:

$$
\begin{aligned}
& q-K z-L z^{\prime}+\left[\left(K^{2} z+L^{2} z^{\prime}\right) / q\right](3-q)=0, \\
& q=\left(4 K^{2} z+4 L^{2} z^{\prime}\right) / q,
\end{aligned}
$$

which, of course, reduce to (21) for $L=0$.

Equations (31) allow us to determine $q_{\mathrm{c}}$ as a function of $L, z$ and $z^{\prime}$, for a given lattice. When considering negative $L$, one should not allow the ratio $|L| / K$ to be too big, since otherwise the ferromagnetic ground state could be destroyed. The effect on $q_{\mathrm{c}}$ of a small perturbation in the Hamiltonian can be studied most easily by linearising (31) in $L$. For a $d$-dimensional hypercubic lattice $\left(z=2 d, z^{\prime}=2 d(d-1)\right)$ we thus obtain:

$$
q_{\mathrm{c} 1}(d, L)=7-2 \sqrt{2 d}-8 d(d-1) L /(7-2 \sqrt{2 d})+\mathrm{O}\left(L^{2}\right),
$$

which clearly shows that a negative $L$ determines an increment, whereas a positive $L$ determines a decrement of $q_{\mathrm{c}}$. This qualitative effect is in agreement with the results of Monte Carlo studies (Fucito and Vulpiani 1982).

\section{Discussion and conclusions}

The $q_{\mathrm{c}}(d)$ curves obtained from our simple reaction field calculations are rather qualitative and still far off known or conjectured values. They do not reproduce the expected limit property $q_{\mathrm{c}} \rightarrow \infty$ when $d \rightarrow 1$, neither are they in agreement with $q_{\mathrm{c}}(2)=4$ or $q_{\mathrm{c}}(4)=2$. Our results demonstrate, however, that one may get away from the trivial mean field prediction by using the conditional probabilities (9), even in their most simple approximation. The larger cluster gives somewhat better results at lower dimensions. More sophisticated approaches along the same lines can be expected to reproduce much more accurate results. In particular, by using the specific lattice structure, as has been done e.g. for the Ising model (Dekeyser and Halzen 1969), one should be able to reproduce better the dimensionality dependence of $q_{\mathrm{c}}$, which is only simulated here through the $z$ dependence.

Our calculation in $\S 5$ deserves, in our opinion, particular interest. It is concerned with the important non-universal character of $q_{\mathrm{c}}$. Despite its simplicity, our calculation gives a result in qualitative agreement with Monte Carlo experiments. To our knowledge, up to now there has been no alternative approximate way of attacking this problem (Saito 1982), and further efforts along these lines could be revealed to be fruitful.

The problem of distinguishing between first- and second-order transitions is a fundamental and general one. Very often it turns out to be extremely difficult to solve this problem, even with the most sophisticated approximation methods, like the renormalisation group. On the other hand, in the case of the Potts model, the existing real space renormalisation group calculations seem to give a too universal character to $q_{\mathrm{c}}$ (Nienhuis et al 1981). In our opinion, it is thus important to have shown in a specific example that it is possible to have non-trivial predictions, even within a mean 
field type of approach, when one takes into account reaction field effects. This is especially remarkable when we realise that, within a classical context, other improvements of the mean field approach, like the Bethe and similar approximations (Domb 1960 ), all lead to the trivial prediction $q_{\mathrm{c}}=2$. Concerning this point, we would like to mention that a criterion has recently been proposed for distinguishing between continuous and discontinuous transitions, based on the comparison of different meanfield-like approximations (Livi et al 1982).

\section{Acknowledgment}

AM and ALS acknowledge stimulating discussions with M Droz and G Parisi.

\section{References}

Baxter R J 1973 J. Phys. C: Solid State Phys. 6 L445

Brout R and Thomas H 1967 Physics 3317

Burkhardt T W, Knops H J F and Den Nijs M 1976 J. Phys. A: Math. Gen. 9 L179

Dekeyser R and Halzen F 1969 Phys. Rev. 181949

Domb C 1960 Adv. Phys. 9149

Fucito F and Parisi G 1981 J. Phys. A: Math. Gen. 14 L499

Fucito F and Vulpiani A 1982 Phys. Lett. 89A 33

Harris A B, Lubensky T C, Holcomb W K and Dasgupta C 1975 Phys. Rev. Lett. 35327

Kihara T, Midzuno Y and Shizume T 1954 J. Phys. Soc. Japan 9681

Livi R, Maritan A, Ruffo S and Stella A L 1983 Phys. Rev. Lett. 50459

Mittag L and Stephen J 1974 J. Phys. A: Math., Nucl. Gen. 7 L109

Nienhuis B, Berker A N, Riedel E K and Schick M 1979 Phys. Rev. Lett. 43737

Nienhuis B, Riedel E K and Schick M 1981 Phys. Rev. B 236055

Onsager L $1936 \mathrm{~J}$. Am. Chem. Soc. 581486

Potts R B 1952 Proc. Camb. Phil. Soc. 48251

Priest R G and Lubensky T C 1976 Phys. Rev. B 134159

Saito Y 1982 J. Phys. A: Math. Gen. 151885

Wu F Y 1982 Rev. Mod. Phys. 54235 